$\mathbb{T}$ periodica polytechnica

\author{
Transportation Engineering \\ $39 / 2(2011) 55+62$ \\ doi: 10.3311/pp.tr.2011-2.02 \\ web: http://www.pp.bme.hu/tr \\ (c) Periodica Polytechnica 2011
}

RESEARCH ARTICLE

\section{Information system for road infrastructure booking}

\author{
Tamás Soltész / Miklós Kózel / Csaba Csiszár / Tamás Centgráf / Balázs Benyó
}

Received 2010-09-06

\begin{abstract}
Booking of road infrastructure is a tool for management of „stresses" between traffic demand and available infrastructure capacity (supply). In this course entitlement to use of certain network elements (lane, zone, and parking lot) can be purchased. It can be applied at those network elements where traffic jams are generated regularly and travel time is unpredictable. Charges for use of infrastructure are proportional to the number of bookings. Its extent influences both travel mode and vehicle choice. "Intervention” in traffic flows is wanted, therefore its analysis and control require the use of knowledge in the following fields: transport network planning, traffic technology and transport informatics; in a system-and process-oriented approach. Model of this complex transport system has been built up; the operations are influenced by dynamic information.
\end{abstract}

\section{Keywords}

road transport network model $\cdot$ route choice $\cdot$ booking $\cdot$ road charge $\cdot$ traffic control.

\section{Tamás Soltész}

Department of Transport Technology, BME, H-1111 Budapest, Bertalan Lajos u. 2., Hungary

e-mail: soltesz@kku.bme.hu

\section{Miklós Kózel}

Department of Transport Technology, BME, H-1111 Budapest, Bertalan Lajos u. 2., Hungary

e-mail:kozel@kku.bme.hu

\section{Csaba Csiszár}

Department of Transport Technology, BME, H-1111 Budapest, Bertalan Lajos u. 2., Hungary

e-mail: csiszar@kku.bme.hu

\section{Tamás Centgráf}

Department of Transport Technology, BME, H-1111 Budapest, Bertalan Lajos u. 2., Hungary

e-mail: tamas.centgraf@altacom.hu

Balázs Benyó

Department of Transport Technology, BME, H-1111 Budapest, Bertalan Lajos u. 2., Hungary

e-mail: bbenyo@iit.bme.hu

\section{Main idea and objective of road infrastructure book-} ing

Booking of road infrastructure is a tool for management of „stresses" between traffic demand and available infrastructure capacity (supply). In this course entitlement to use of certain network elements (lane, zone, and parking lot) can be purchased. It can be applied at those network elements where traffic jams are generated regularly and travel time is unpredictable. Charges for use of infrastructure are proportional to the number of bookings. Its extent influences both travel mode and vehicle choice.

Vehicles' space-demands are caused by their moving and parking. The whole time consumption of locomotion is affected by travel and parking (searching for parking lot) times. The aim is to offer predictable travel time for drivers. Toward it, booking procedure is suggested for both sub processes (travelling, parking), or rather for both types of network elements (routes, parking lots). In this way (in a wider interpretation) "booking of road infrastructure" is realized.

Trip-booking belongs to "hard tools" of traffic management, namely the rules introducing simultaneously are valid for all participants in road traffic (users of controlled and uncontrolled elements of the transport network). Only registered drivers can make booking. Since number of vehicles is limited on controlled network elements per time unit (and demands often exceed it), mobility demands of users without booking (permission) are also to be managed in an alternative way.

There is a quasi contrast between individual character of road transport and controlled traffic. Traffic technological aim is avoidance of instable traffic situation. Forasmuch only a segment of the entire road traffic will be controlled in this manner that is why particular attention should have been paid to the "joints" of traffic flows on controlled and uncontrolled network elements as well as in parking zones. The suggested system will "cover" the following cases and their combinations:

\section{Management of movements}

- booking of routes (nodes and links),

- granting permission to drive in a certain area (zone), 
Booking of routes can be particularly realized on multilane roads, because uncontrolled traffic should be kept in most cases. Number of drive-in permissions in a residential area correlates to the number of available parking spaces.

During elaboration of this concept a comprehensive theoretical knowledge has been developed. However, several issues of practical implementation also have been revealed. Detailed recommendations for information system aiding road infrastructure booking have been devised.

\section{Main objectives of the concept:}

- Preferring controlled traffic to uncontrolled traffic within individual motorised transport; at the same time the other transport modes (non-motorised and public transport) henceforward have higher priorities.

- In consequence of faster travelling reduced time consumption (which can also be converted into saved monetary units), as well as reduced environmental load.

- "Smoothing" of peak demands in time. Alteration of travel habits (e.g. passengers plan their trips for a later time) [3].

- Influence on demands in space (e.g. with charge rates). Fees for road booking in inner urban areas will meet presumably smaller social resistance than toll for city road use in general, because in our solution drivers get calculable travel time in exchange for the fee.

- Encouragement of public transport use at travellers who are "crowded out" from booking. Initial introduction of booking system is advisable at the locations where public transport is attractive (e.g. metro, $\mathrm{P}+\mathrm{R}$ ).

- Return of money - income of booking system - for transport purposes; however, the original aim is to make capacity exploitation of road infrastructure calculable.

- Determination of charges proportional to "measure" (distance, time consumption) of booking.

- Stimulation for higher vehicle occupation (for more efficient space utilization) by application of preferences.

- Preference of environment friendly vehicles.

- Making controlled traffic flows also on bus lanes permitted (common use); in this way reducing of unexploited capacities (when buses run infrequently).

\section{Summary of prior lane management strategies}

One way of the management (control) of road traffic demand is recommendation, when information on current traffic conditions is given to drivers and routes with lower traffic are advised.

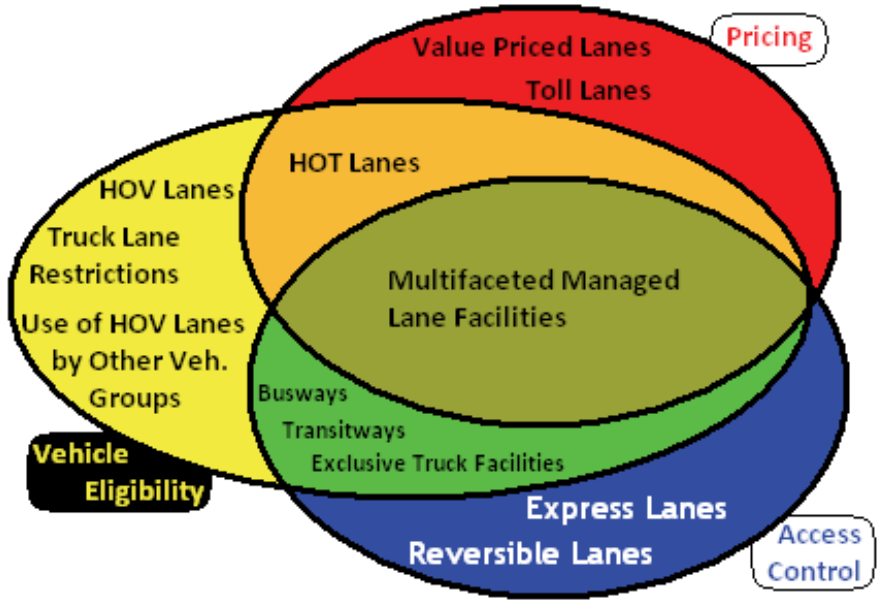

Fig. 1. Lane management strategy complexity [4]

Further methods are the introduction of limitations or pricing. These strategies are summarized on Fig. 1.

Obligatory fees occur on many elements of the network, but in most cases, the objective is not avoidance of congestion but cover of operation costs. There are also solutions where higher prices are applied on certain roads (or particular lanes of them) resulting lower traffic. Other forms of road pricing are congestion charges (e.g. London, Stockholm, Singapore). In zones like these not only certain types of vehicles are forbidden from traffic flow but whole traffic is restricted. There are many forms of vehicle eligibility. For instance, prohibition of slow vehicles and preferring high occupancy vehicles (HOV) enable to achieve larger traffic outputs (passenger kilometres). With access control different traffic layers can be separated. For example, dissociation of short- and long-distance traffic flows decreases the disturbance caused by interaction of vehicles; while reversible lanes help distribution of road capacities to match the demands. Public transport is a separate layer of traffic and a special vehicle type at the same time; hence its separation from uncontrolled traffic is reasonable from several aspects [4].

With the combination of different strategies, control can be made more sophisticated. A typical example for this is the principle of HOT (High Occupancy \& Toll) lanes, where lowutilized HOV lanes are used by vehicles with lower occupancy for fee. The reason for this way of operation is that the amount of vehicles can be influenced by the number of passengers in very large "steps", while a more precise control can be realized with charges. Many existing tariff systems are really complex, moreover, in the United States there are some places where the rate of charge varies dynamically depending on the number of vehicles. (Drivers are informed about the actual price by variable message signs.)

A brand new way of control is the requirement of preliminary booking to use a network element. Although this method is widespread in passenger transport, its use in road traffic is only theoretical. Even in the literature, booking is mentioned only for long-distance traffic [2, 3, 5]. The reason for this is that in- 
formation technology tools required for trip booking have been developed only in the last years. However, the investigation of this control method is relevant nowadays, because trips can become more calculable, and larger networks can be handled with booking. The best results can be achieved if booking is applied along with the methods mentioned above.

\section{General model of transport network and traffic pro- cesses}

For realization of trip-booking and related routing system modelling of transport network is needed. In this graph model, nodes match points of direction-change (turn-off or joining), while edges are directed links which connect nodes and conduct traffic flows. So we have broken up traffic junctions to branches, considering turning opportunities. Controlled zones and heads (gates) of links conducting to uncontrolled areas are additional nodes. We recommend a graph in which the number and structure of edges are constant, but the resistance of them is varying in time.

The network graph consists of three types of elements:

- Road-sections (controlled and uncontrolled),

- Zones (controlled and uncontrolled),

- Parking facilities (only controlled ones are concerned).

A critical point of symbolization is comparison the number of bookings with the capacity of elements. For this, whole booking time is cut into time slots (marked with $i$ ) and capacities are examined in each slot separately. Because of uncertainty of travel time, it can cause problems when a vehicle arrives to a section at the border of time slots. In such situations this vehicle is "distributed" between the slots in the ratio of probability of appearance.

Calculability of travel time on controlled network elements also depends on their adequate contact with uncontrolled elements. A booking will be calculable only if it begins and ends on points that are controlled or situated in areas where no congestions are (generally).

Dynamic resistance of graph edges can be determined by charge $\left(C_{x}^{i}\right)$ and travel time of each element $(x)$. Charge is also transformed into time by means of a user-dependent time equivalent $\left(\tau_{u}\right)$. Travel time on sections and junctions can be calculated by previous, historical data (considering time of day and day of week) - marked by $t_{x}^{i, h}$. In zones and parking facilities, travel time consists of two parts. The first represents the travel to the border of the zone or parking, while the second stands for the movements inside the element (this is a constant depending on the size of element). Hence, resistance $\left(t_{x}^{i}\right)$ of element $x$ in time slot $i$ :

$$
t_{x}^{i}=t_{x}^{i, h}+\frac{C_{x}^{i}}{\tau_{u}} \quad \text { while } B_{x}^{i}<B_{x}^{i, \max }
$$

- $t_{x}^{i}$ - resistance of element $x$ in time slot $i$,
- $t_{x}^{i, h}-$ historical travel time,

- $C_{x}^{i}$ - charge,

- $\tau_{u}$-user-dependent time equivalent,

- $B_{x}^{i}$-number of bookings,

- $B_{x}^{i, \max }$ - predefined maximum number of bookings.

If the number of bookings $\left(B_{x}^{i}\right)$ reaches a predefined maximum value $\left(B_{x}^{i, \max }\right)$, resistance of the edge becomes infinite large, so additional vehicles cannot be admitted to drive in. In case of roads, junctions and parking facilities, this limit is primarily determined by road parameters, green times or number of parking lots. In zones - because transit is prohibited - capacity can be regarded as the number of parking lots. On parking elements, bookings can also be "open-ended", therefore leaving time of some vehicles is not known in advance. Reissue of these lots can be made with a delay, which reduces exploitation. The capacity of edges has to be reduced by an environmental factor $\left(e_{x}^{i}\right)$ and a spare factor $\left(s_{x}\right)$. Some redundancy is considered, but we do not deal with extreme traffic situations caused by technical breakdowns or human faults. So the maximum of permissions:

$$
B_{x}^{i, \max }=N_{x}^{\max } e_{x}^{i} s_{x}
$$

- $B_{x}^{i, \text { max }}$ - predefined maximum number of bookings,

- $N_{x}^{\max }$ - capacity of network elements,

- $e_{x}^{i}$ - environmental factor,

- $s_{x}$ - spare factor.

Introduction of such a system will have a great effect on traffic. Realignment of traffic should be examined with (macroscopic and microscopic) simulation. Simulation is a part of planning procedure which consists of transport network modification as well as determination and evaluation of expectable effects. The procedure is repeated until the most appropriate version is found. (It is possible by installation of a controlled element, the traffic on an uncontrolled element grows to an unmanageable level, so further versions have to be examined.) This series of steps is also able to demonstrate the effects of different states and partial results, say step-by-step implementation (for example, the stages of extending controlled network).

\section{General features of information system}

Intelligent Transport Systems can aid smooth exploitation of available capacities without significant investments into transport network. They promote traffic redistribution both in time and space as well as reduce risk of accidents caused by grown traffic. Fig. 2 shows structure of the solution that includes booking of routes.

Operation can be broken down into the following most important sub processes (as the figure shows): 
1 Drivers ask for permission to use the controlled network (providing their spatial, temporal and other parameters). Central data processing system (may) grant it on the basis of actual road status, environmental, traffic, etc. features and information coming from related traffic control (management) systems as well as purpose of traffic management.

2 Controlled network elements and vehicles running on it are permanently monitored. Drivers without permission or exceeding its scope (either in space or time) are selected and taken under sanctions.

3 Data of road situations, environment, traffic, etc. are collected with a certain sampling time. These data influence parameters of permissions to be issued.

4 Issued permissions have effect on traffic layers. Drivers with permission get their destinations in a predictable travel time. On uncontrolled network "additional" congestions may occur (periodically), namely vehicles crowded out from controlled network elements appear on the rest (still available) routes. Therefore some travellers shall choose other, alternative (e.g. non-motorised or non-private) transport mode.

Operation consists of data collection, processing and consumption as well as feedback. Elements of data collection (traffic loops, detectors, weather stations, video cameras, etc.), telecommunications subsystem, units of data storage, processing, evaluation, subsystem of interventions as well as human "components" (dispatcher) are all required for execution of tasks. Many operations - but not all - can be automated. System components that operate in an intelligent way should also be implemented; namely algorithms substitute human mind during frequent tasks; so thus eliminating also failures. The suggested information system manages real-time data predominantly automatically; interventions are based on actual traffic parameters and considering similar traffic situations in the past. Information and fee collection also belong to traffic control (management) [1].

In this control structure "sampling-time" is a fundamental issue. This time interval has coherence to the velocity of the simulation and capacity of all telematics components. Minimum and maximum values can be determined for sampling-time.

\section{Structure of the information system}

Structure and operation of the integrated system are demonstrated on Fig. 3

\section{Telematics background of road infrastructure booking}

Information system of booking consists of central servers (with database) and interactive user terminals (with several functions). These elements are connected by internet data communication technology (with web-based interfaces). Driver (vehicle) terminals' abilities for data storage, process, input and output depend on configuration and expected functions. Even in most advanced cases the "thin-clients" (focused on positioning and navigation operations) are used with preference. As many as possible information procedures of routing, booking and inspection (comparison) functions should be allocated at central servers. It is particularly important at the so called "global optimum" criteria. Clients of booking information system are partially non-bound for terminal use. This group uses booking service occasionally (e.g. foreign drivers) or very frequently (e.g. pizza couriers with monthly passes). In these cases all information procedures relating to booking system are very simplified.

Data transmission is executed by high-speed radio network. Data are processed by high-capacity computers (with "warm backup") in the centre. Data communication with the "conventional" traffic control centre will have more and more significant role in the future.

Devices for collection of information about controlled network elements

Loop-detectors measure parameters of traffic flows. Weatherstations give meteorological data; these stations measure not only the ,conventional” parameters, but the features (e.g. temperature) of the road surface and its surroundings (e.g. sight reach) as well. Basically, automatic data collection is missing on uncontrolled network elements.

\section{Devices for monitoring existence and validity of permis- sions}

Installed video cameras with "picture evaluation" software are primarily suitable for monitoring of permissions. They compare monitored traffic (after recognition of number plates) and data of booking, using data of "background database". Technology of vehicle monitoring and tracking is suitable for control of permissions' validity (spatial and temporal). For this realization the appropriate terminal should be in service at all registered clients.

\section{The operation of the information system}

Registration of drivers (vehicles)

Two groups of booking clients can be made:

- those who have telematics device on board (involved in vehicle tracking),

- those who don't have telematics device on board, like occasional users, users with passes (representing a lower rate and a higher charge) and residents. About $10 \%$ of the capacity could be resorted this way.

More user profiles could be created by the propulsion system of the vehicle (e.g. gas, electrical), the characteristics of passengers (e.g. disabled) and in case of other public vehicles (e.g. taxis) etc.

Registration is compulsory for each user. Customer service points with personnel support (e.g. petrol station) are available for registration, where personal and vehicle data could be 


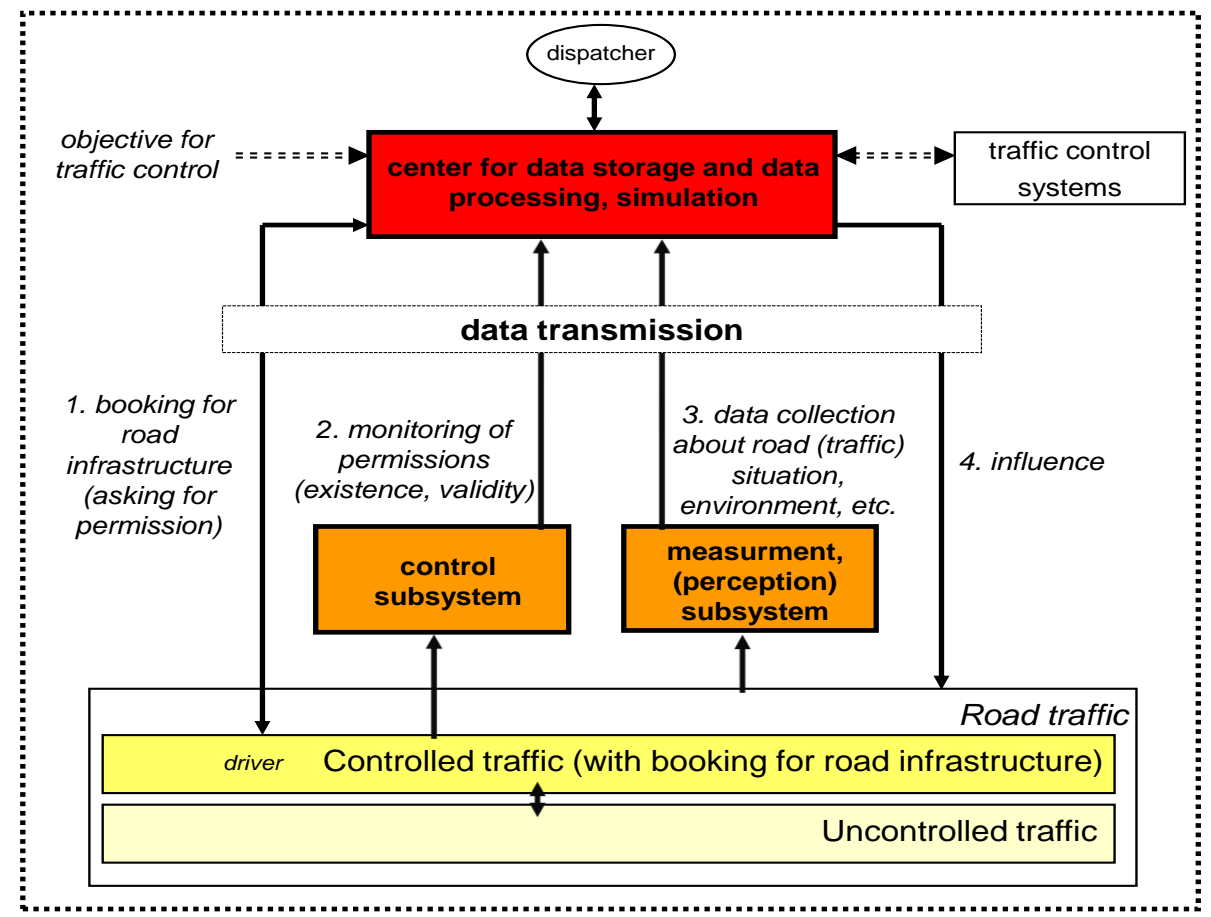

Fig. 2. Structure of intelligent transport system that includes booking for road infrastructure

recorded (verification also can be done here). Occasional users and users with passes - initially - are allowed to pay charge in cash before travelling. A code for identification is provided for those who are registered and have on board device.

In case of occasional users, personnel do the routing and booking (granting so-called daily permissions). Users with passes are allowed to use any controlled network elements except for parking lots - without booking. Residents in controlled zones are also allowed to enter without booking, however, drivers without garage have to book their parking lots in advance (a reduced charge is granted). Their registration is made simultaneously with the issue of permissions to enter.

\section{The role of routing in booking process}

Starting and destination points, departure time and the latest arrival time as well as time equivalent $\left(\tau_{u}\right)$ are given by the users. Based on these data as well as vehicle parameters (e.g. occupancy, propulsion system) and network parameters (e.g. air pollution levels on links) a few appropriate - bookable - routes are offered for users (as well as booking charges).

\section{Conditions, validity and issue of booking permissions}

Route selection is followed by booking of connected network elements (and the issue of booking permissions). Permission is valid for the selected network elements if travel begins in the defined departure time interval (slot $i$ ). Each traffic layer can be filtered out at the issue of permissions. For example, in urban areas heavy vehicle traffic can be limited (in time or the number of vehicles). On the other hand, priority can be granted for layers e.g. electrical vehicles. These facts might influence the charge. (Downtown loading stands could be involved into the booking system in the future). In case of vis maior - when booking cancellations are necessary - certain layers can be preferred.

In the time interval before the defined departure time (in slot i1) booking is not available (neither cancellations are possible) because the booking system operates with the aforementioned cycle time. Fee collection is automatically executed at the beginning of the defined departure time interval. The transaction is accomplished through a pre-paid "virtual wallet" instead of directly from a bank account (access problems, legal issues). The owners of parking lots in controlled zones are allowed to enter without any permission in all cases.

\section{Factors influencing rate of booking charge}

Booking charge is the sum of separately defined charges for booked network elements.

$$
C=\sum_{x} C_{x}+C_{z}+C_{p}
$$

- $C$-total booking charge,

- $C_{x}$ - booking charge of links,

- $C_{z}$ - booking charge of zones,

- $C_{p}$ - booking charge of parking lots.

In case of links the following factors can influence the rate of booking charge:

$$
C_{x}=C_{0_{x}} l_{x} p
$$

- $C_{0 x}$ - basis booking charge, depending on e.g. time of day (during low traffic periods, e.g. at night between $10 \mathrm{pm}$ and 5 am, controlled routes can be used free of charge),

- $l_{x}$ - length of routes and other network parameters, 


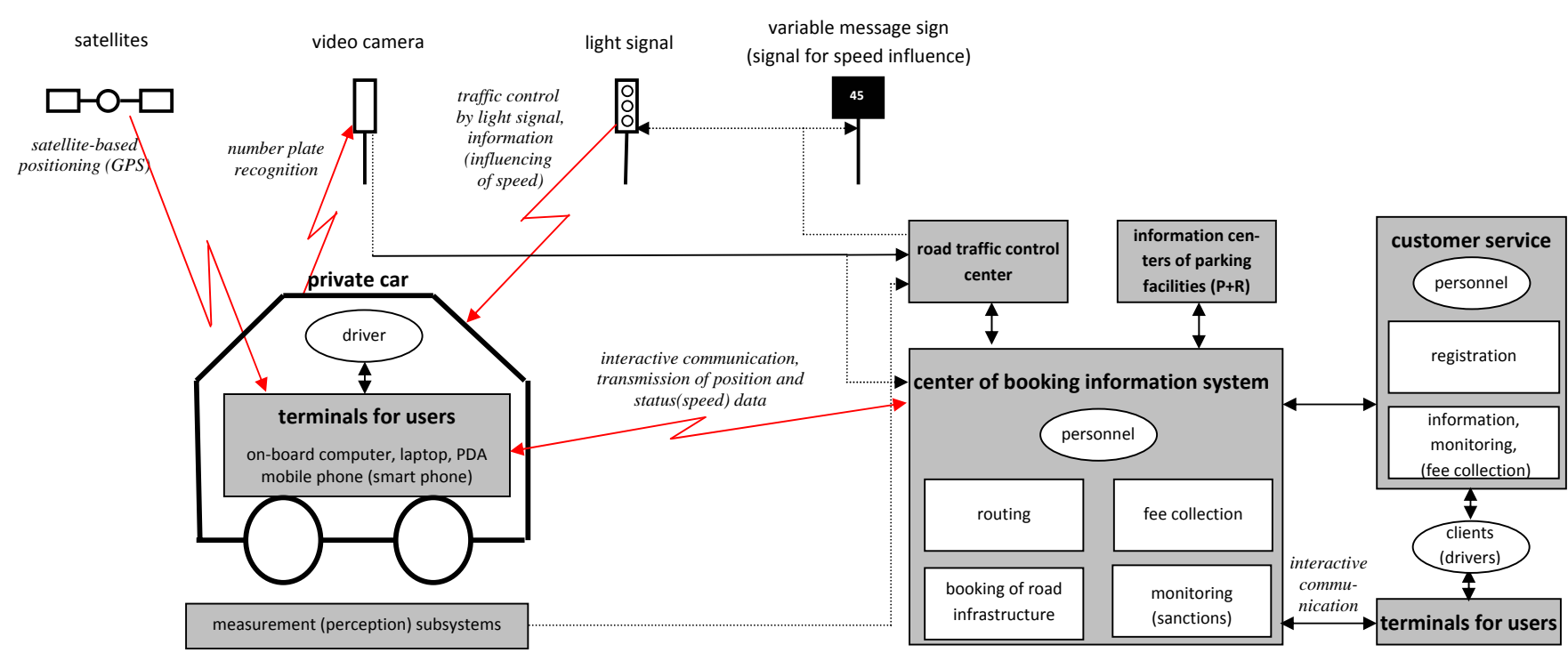

Fig. 3. Structure of the information system for road infrastructure booking

- $p$ - number of passengers or other vehicle parameters.

(If the rate of booking charge is influenced by the number of bookings in the time interval of arrivals onto links as well, the use of an additional exploitation multiplier is needed.)

In case of zones following factors can influence the rate of booking charge:

$$
C_{z}=C_{0_{z}} t_{z} d
$$

- $C_{0 z}$ - basis booking charge, depending on e.g. time of day (similar to links, e.g. at night between $10 \mathrm{pm}$ and $5 \mathrm{am}$ entrance is free) or location of zones in the city structure (different basis charge according to each categories),

- $t_{z}$ - duration (linearly varying charges according to the duration of use),

- $d$ - discounts (e.g. for residents, number of vehicles per household etc.).

In case of open-ended booking (namely drivers do not know the duration of stay) value of $t_{z}$ is determined later, according to the exact time of leaving provided by vehicle tracking. Thus, higher basis charge $\left(C_{0 z}^{\prime}\right)$ is taken into account. In case of zones payment is associated with entrance (leaving is free of charge).

In case of parking the following factors can influence the rate of booking charge:

In case of parking facilities booking charge is defined according to the tariff of the operator $\left(C_{p}\right)$. In any other cases (e.g. parking along shoulder):

$$
C_{p}=C_{0_{p}} t_{p}
$$

depending on:

- $C_{0 p}$ - basis booking charge, depending on e.g. time of day (free of charge periods) and different categories (e.g. location),

- $t_{p}$-duration of use.
It is practical to implement a charge varying progressively according to time; thus preferring shorter shoulder parking time. Transport policy objectives may also be considered at determination of booking charges. Charges have to be socially adopted thus exact communication and the presentation of available benefits are required.

Features of constant, progressive and degressive charges depending on the exploitation rate (variation modes of the exploitation multiplier)

- constant:

The idea of using constant charges is based on the assumption that same service level of traffic could be granted for all users (whenever they enter) - under the capacity limit.

- progressive (increasing):

Earlier bookings could be inspired this way. However, the difference between the lowest and the highest charge cannot be multiple (a few charge levels should be evolved).

- degressive (decreasing):

At use of decreasing charges proportionately to the number of bookings it is assumed that higher exploitation rate causes more likely higher traffic chaos. This solution is not really appropriate because of the possible 'hesitation of drivers'.

\section{Information of drivers about the booking system}

Drivers should be informed about controlled network elements by large-scale, visible roadside signposts containing static information. Registered (regular) users get personalized (dynamic) information on an interactive on-board device. In case of "non required on-board device" users preliminary information is important, because dynamic information cannot be sent to them during their trip. In addition, variable message signs along the road can give complementary information as well (e.g. recommended speed). 


\section{Monitoring of permissions}

The best solution for monitoring the existence of permissions and temporal as well as spatial validity is the combination of following methods:

Based on installed equipments: cameras observe links, connection points of zones and parking lots. In case of vehicles without permission the evaluation system warns by signalization.

Based on vehicle tracking: Data link works between the center of booking system and on-board or personal units, thus, permissions can be monitored easily by a frequent, short query interval. In case of vehicles without permission the evaluation system warns by signalization. (Vehicle tracking by FCD - Floating Car Data method could provide information about network elements, e.g. average speed.)

Factors that affect penalty charges of illegal use; additional sanctions

Illegal users are those who:

- are registered and have booking permissions but use routes and intervals in a non-appropriate way,

- are not registered and do not have booking permission but use controlled network elements.

Rate of penalty depends on:

- frequency of perpetration, recurrent or occasional offence,

- parameters of illegal use (e.g. the length of using controlled elements).

Sanctions are needed in case of timeout. Late departure (one or two slots delay) bring forth low penalty. In case of punctual departure but involuntary timeout (impossible leaving of booked network element) penalty is applied. Sanctions can be taken even if the occupancy of vehicle differs from the preliminary parameter and that is not notified (before charging). Further sanctions can be used, for example: restriction of booking or even exclusion.

\section{Management of "vis maior" situations}

It is not possible to prepare for vis maior therefore the object is handling the actual traffic situation. The main procedures are:

- abort issuance of further permissions,

- cancel of already issued booking permissions whether the driver has not departed yet (compensation is needed),

- for those who have already departed information and suggestion of alternative (controlled) routes are necessary, if possible (partial compensation is granted).

\section{Introduction of the booking system}

New network elements can be added step-by-step to the tripbooking system. Initially, the system should be introduced in a few locations and with simplified technical background, but later it can be improved both in space and technology. Even in the "basic version", it is important to pay attention to the joints of controlled and uncontrolled elements. Therefore we suggest an initial configuration that consists of a long section of a multilane road passing through the city diametrically and some zones in the centre. In this case controlled lanes can be leaved only toward controlled or rarely congested elements. However, the low number of controlled elements makes possible to simplify the system in several ways.

Possibilities for simplification:

- More simple informatics background (e.g. phone-based system),

- At few elements, routing can be skipped,

- Discarding open-ended bookings,

- Enforcement only with cameras, without on-board devices.

Possibilities for improvement:

- Using more specified network model,

- Introduction of location-based charge system (approx. 5-6 charge categories for controlled road sections, depending on their location, traffic, sensibility, etc.),

- Refinement of discount-system (lower prices for environment-friendly and smart vehicles).

\section{Conclusions}

By the presentation of this conception we would like to draw attention to a novel way of managing road traffic demands that exceed capacities. Even in this theoretic elaboration, several problems waiting for solution have arisen, but the real challenge will be the appliance. On each network element detailed examinations will be necessary before implementation, because while vehicles taking part in booking are preferred, others using uncontrolled elements — can get disadvantage. The basic requirement of appliance is a positive balance of benefits and drawbacks on the whole road system.

Altacom Itd. and $\mathrm{mS}$ Systems Itd. have been developing a working system on the basis of the presented conception. 


\section{References}

1 Csiszár Cs., Nagy települések személyforgalmának integrált dinamikus irányítása telematikai eszközökkel, Városi Közlekedés 44 (2004), no. 2, 8497.

2 Edara P, Teodorović D, Model of an advance-booking system for highway trips., Transportation Research Part C , posted on 2008, no. 16, 36-53, DOI 10.1016/j.trc.2007.06.004, (to appear in print).

3 de Feijter R, Evers J M, Lodewijks G, Improving Travel-Time Reliability by the Use of Trip Booking, IEEE Transactions on Intelligent Transport Systems 5 (2004), no. 4, 288-292, DOI 10.1109/ITSC.2003.1251949.

4 US. Department of Transportation Federal Highway Administration, 2004. Managed Lanes - A Cross-Cutting Study.

5 Wong $\mathbf{J}$ T, Basic concepts for a system for advance booking for highway use, Transport Policy 4 (1997), no. 2, 109-114, DOI 10.1016/S0967070X(97)00006-1. 